\title{
The two-dimensional cutting stock problem within the roller blind production process
}

\author{
E.R. de Gelder, A.P.M. Wagelmans * \\ Econometric Institute Report EI 2007-47, October 2007
}

\begin{abstract}
In this paper we consider a two-dimensional cutting stock problem encountered at a large manufacturer of window covering products. The problem occurs in the production process of made-to-measure roller blinds. We develop a solution method that takes into account the characteristics of the specific problem. In particular, we deal with the fact that fabrics may contain small defects that should end up with the waste. Comparison to previous practice shows significant waste reductions.
\end{abstract}

Keywords: cutting, two-dimensional cutting stock problem, trim loss,

\section{Introduction}

The research reported on in this paper has been carried out for a large manufacturer of window covering products. To protect its interest we will refer to the manufacturer as company X. One of X's window covering products are madeto-measure roller blinds. As with almost any product in any company, cost reduction is a major issue in the roller blind production process. A large part of the cost of a roller blind is determined by the materials used, in particular

*Erasmus University Rotterdam, Econometric Institute, P.O. Box 1738, 3000 DR Rotterdam, The Netherlands, E-mail: wagelmans@few.eur.nl 
the tubes and the fabrics. Consequently, reduction of the inevitable waste of material during production is favorable.

Roughly speaking, one could say that a roller blind consists of four main elements: a lower tube to keep the roller blind down, an upper tube on which the roller blind is fixed, a piece of fabrics in between, and a mechanism to operate. The required fabrics are cut from large rolls of fabric and assumed to be available in unlimited quantities.

Customers have orders consisting of one or more items. Each item has its dimensions and a required quantity associated with it. At X, the production of roller blinds is order-based, i.e., the dimensions and fabric design are specified by the customer. This means that all orders are unique, which makes it impossible to use standardized patterns to cut the fabrics. Instead, one should minimize the waste for each new batch of orders, i.e., one has to solve a two-dimensional cutting stock problem (2D-CSP).

In 1965, Gilmore and Gomory [10] proposed an extension to their famous "delayed pattern generation"-technique for the one-dimensional CSP $[8,9]$ in order to cope with CSP's of two and more dimensions. They considered the problem of cutting small rectangles out of a supply of large stock rectangles using as few rectangles as possible. Although common structures can be found in real-life cutting problems, they often differ significantly with respect to certain goals or constraints [3]. Unsurprisingly, the number of solution techniques that have been developed throughout the years is large.

In our opinion there are seven characteristics that determine which solution techniques can be applied: difference of sheet sizes, relative order dimensions, multiplicity, size, orthogonality, guillotine cutting, and item orientation. This list has some overlap with Dyckhoff's classification [6], however, we think it better covers the relevant aspects encountered when analyzing the problem.

The cutting of the fabrics for roller blinds can be described as cutting relatively large rectangles with low multiplicity from a large roll of fabrics thereby minimizing the waste. The size of the problem is relatively small (approximately 20 to 25 items to cut per batch) and the cuts are restricted to be orthogonal and guillotine. In principal, the items do not have a fixed orientation (i.e., a rotation of 90 degrees is permitted). However, some fabrics have a certain design which does not permit a rotation. Furthermore, there are fabrics having 
a certain design that allows rotation of an item if and only if all items of the same customer are also rotated. Finally, the fabrics contain small defects, i.e., small areas which cannot be used for production. Fortunately, the exact locations of these defects are known in advance which means that we can use this information during the determination of a cutting plan.

Most articles found in literature consider problem instances based on Gilmore and Gomory's problem (for example $[2,13,16]$ ), which means that it is assumed that all stock sheet sizes have the same dimensions (or a few different standard dimensions). Furthermore, the solution technique of solving the Linear Programming (LP) relaxation and transforming the resulting fractional solution to an integer one, only results in high quality solutions if the multiplicity of the items is high [5]. Due to the fact that we can unroll the fabrics up to any length (with a maximum determined by the physical length of the cutting table), we could say that the problem we will be considering consists of sheets with variable height. Furthermore, due to the fact that most customers buy roller blind for private use, the multiplicity of the items is low (on average approximately 2). All together, this means that the Gilmore and Gomory-based solution techniques are not applicable to our problem. The use of cutting tables restricts the cuts to be orthogonal which is a common assumption. However, it should be noted that De Cani [4] has shown by a simple example that the restriction of orthogonal cutting patterns can prevent the optimal arrangement from being obtained. The cutting table also restricts the solution space to include only guillotine patterns. For some recent non-guillotine solution approaches the reader is referred to $[14,15]$. How to deal with defects within the 2D-CSP is rarely discussed in literature. Hahn [12] has written an article on this subject. However, she described a solution approach for the 2D knapsack problem, whereas we have have to deal with the 2D-CSP with variable height.

In this paper we present a method, the "Two Stage Heuristic" (TSH), specifically designed to solve the 2D-CSP as just described. In the first stage, discussed in Section 2, cutting patterns (here called "shelves") are selected. The second stage (Section 3) determines a sequence in which the patterns are to be cut. In particular, the aim in this stage is to minimize the additional waste due to defects. We will apply the TSH to an example problem in Section 4 and in Section 5, we will compare the obtained results with previous practice. Section 6 contains concluding remarks. 


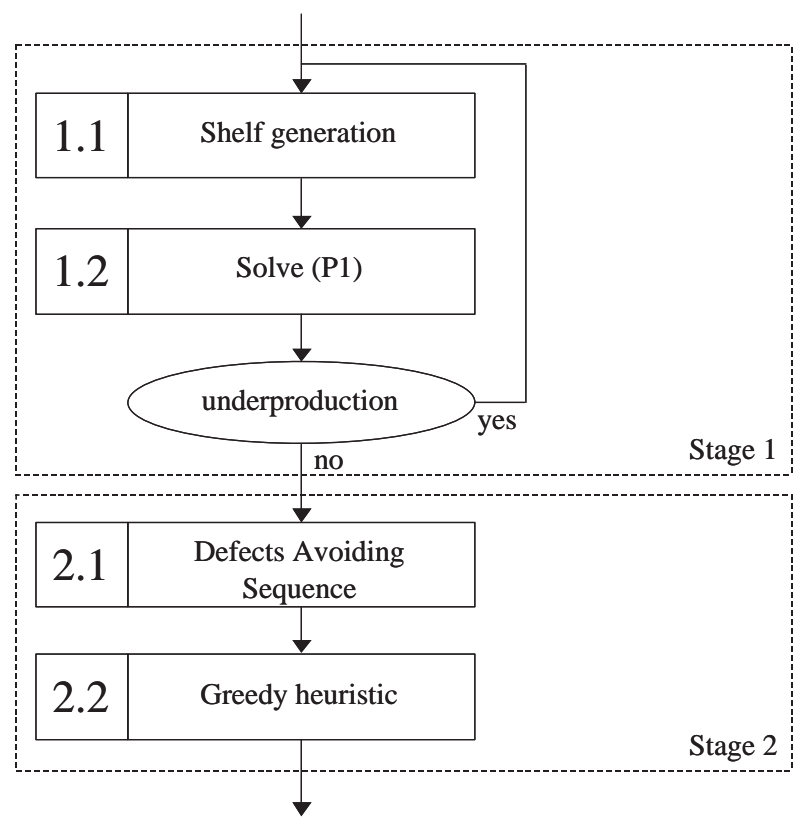

Figure 1: The two stage heuristic.

\section{First Stage}

The first stage of the TSH is based on Gilmore and Gomory's idea [10] of "strips" and "patterns" (here called "bases" and "shelves"). However, due to the low multiplicity and the variable height of our problem, we will use a different solution technique. Figure 1 gives a schematic representation of the TSH.

The first stage of the TSH is an extension of Goulimis' idea [11] to solve the 1DCSP by enumerating the possible cutting patterns and solving the associated integer program by a combination of cutting planes and branch-and-bound. We will refer to the cutting patterns as shelves. An example shelf is shown in Fig. 2. This shelf is created in two steps. First two bases are created by cuts in the horizontal direction, and subsequently these bases are cut into items. Note that trimming is used, i.e., we allow items with different heights in one base. So we need an additional cut to separate the smaller item ( $B$ in this example).

The objective of the first stage is to create a set of shelves. Although not forced by practice, we will restrict ourselves to two-steps shelves with trimming in 


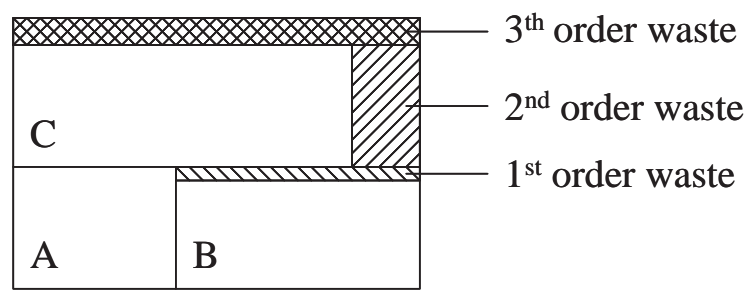

Figure 2: A two-steps shelf with trimming consisting of two bases.

order to limit the number of possibilities. Note that in literature, a two-steps shelf is often referred to as a "two-stage" shelf [1]. To avoid confusion with our two-stage heuristic we prefer to use the term two-steps. We are aware of the fact that by adding the two-steps constraint we might exclude high quality solutions. However, since the relative order sizes are high, the probability of the existence of a feasible three-steps shelf is small. Furthermore, from a practical point of view, a three-steps shelf is even unwanted, since the corresponding increased complexity increases the probability of production failures (at X, cutting is done manually). Finally, it should be noted that even for the relatively small problem instances that we are considering, the number of feasible shelves can be huge. Therefore, we will only take the most promising shelves into account, i.e., shelves that have a relative waste percentage below parameter $\xi$ which is iteratively set during the shelf generation phase. We start with $\xi=1$, i.e., we allow all shelves. As soon as the number of shelves exceeds a certain maximum (600 in our case) we iteratively decrease the value of $\xi$, thereby "throwing away" relatively bad shelves. The goal is to end up with approximately 600 shelves, however, we can only decrease the value of $\xi$ if for each item we will continue to have at least one shelf in which the item is used in rotated position and another one in which the item is used in non-rotated position (assuming that we are using a fabric that allows rotation if and only if all items of the same order are rotated). Note that in case the fabrics allows free rotation or no rotation at all, we only require one shelf in which the item is used. Finally, note that we will not create infeasible shelves, i.e., shelves that internally result in overproduction of a certain item or internally violate rotation requirements.

At the end of this first phase of the first stage (1.1 in Fig. 1) we end up with a set $\mathcal{S}$ of "promising" shelves. Now consider the following problem formulation: 


$$
(P 1): \quad \min _{s \in \mathcal{S}}\left(W_{s}+\epsilon\right) x_{s}
$$

subject to

$$
\begin{gathered}
\sum_{s \in \mathcal{S}}\left(a_{m s}+\hat{a}_{m s}\right) x_{s} \geq q_{m}, \quad \forall m \in \mathcal{M} \\
\sum_{s \in \mathcal{S}}\left(a_{m s}+\hat{a}_{m s}\right) x_{s} \leq q_{m}+O P, \quad \forall m \in \mathcal{M} \\
\sum_{s \in \mathcal{S}} a_{m s} x_{s} \leq\left(1-u_{r}\right)\left(q_{m}+O P\right), \quad \forall m \in \mathcal{M}_{r}, r \in \mathcal{R} \\
\sum_{s \in \mathcal{S}} \hat{a}_{m s} x_{s} \leq u_{r}\left(q_{m}+O P\right), \quad \forall m \in \mathcal{M}_{r}, r \in \mathcal{R} \\
x_{s} \in \mathbb{N}, u_{r} \in\{0,1\}, \quad \forall s \in \mathcal{S}, r \in \mathcal{R}
\end{gathered}
$$

where

$\mathcal{M}$ : set of all items

$\mathcal{R}$ : set of all customers

$\mathcal{M}_{r}$ :set of all items of customer $r \in$ mathcalR

$W_{s}$ : waste of shelf $s \in \mathcal{S}$

$a_{m s}$ : number of times item $m$ is non-rotated in shelf $s \in \mathcal{S}$

$\hat{a}_{m s}$ : number of times item $m$ is rotated in shelf $s \in \mathcal{S}$

$q_{m}$ denotes the required quantity of item $m \in \mathcal{M}$

$O P$ : maximum allowed overproduction

$\epsilon$ : input parameter

$x_{s}$ : number of times shelf $s \in \mathcal{S}$ is used (decision variable)

$u_{r}$ : 0/1-variable; equal to 1 (0) if all items of customer $r$ are (non-)rotated

Before we discuss the model, let us first remark that we have to permit some overproduction in order to ensure feasibility of the problem formulation. This is because we only use the most promising shelves, and therefore feasibility is not automatically guaranteed. How we achieve feasibility will be explained later on.

As a consequence of allowing overproduction, we do not simply minimize total waste in objective function (1). To explain this, consider the situation in which 
a certain shelf has no waste at all. This means that this shelf does not have any contribution to the objective if we would minimize total waste. As a result, its quantity can be increased without deteriorating the objective function value till the maximum overproduction is reached. Because unnecessary overproduction is to be avoided, the objective function coefficients are modified by adding input parameter $0<\epsilon \ll \min _{s \in \mathcal{S}} W_{s}$. The constraints state that production has to satisfy all orders (2) with a maximum on the overproduction (3), and they ensure that all items from the same customer have the same rotation (4)-(5). Furthermore, note that in case that all items can freely rotate or have a fixed orientation, the last two constraints should be omitted. Furthermore, in the case of fixed orientation $\hat{a}_{m s}$ should be omitted too.

As we just argued, we have to permit a certain overproduction to ensure a feasible problem formulation. It is obvious that due to the fact that we only create "promising" shelves feasibility of the MIP formulation $(P 1)$ cannot be guaranteed if $O P=0$. However, recall that for each item there exists at least two shelves: one in which it has been used in rotated position and once in nonrotated position. As a result, it can easily be shown that there exists a finite value of $O P$ for which $(P 1)$ is feasible. Therefore, we will set the value of the maximum overproduction $O P$ iteratively. This means that we first try to solve $(P 1)$ with $O P=0$. If the problem turns out to be infeasible, we will increase $O P$ by 1 and so on till a feasible solution can be computed. The resulting set of shelves $\hat{\mathcal{S}}$ will, in general, have some overproduced items in it. Before proceeding, we will remove the overproduction by removing from $\hat{\mathcal{S}}$ the worst shelf (in terms of relative waste) that contains an overproduced item. We will repeat this step until all overproduction has been removed. Note that we will always end up with at least one shelf since each shelf is feasible by itself. After removing the overproduction, we probably have created underproduction. If so, we will save the obtained set of shelves, adjust the required quantities of the items (these become equal to the under production of the respective items) and repeat the cycle of base \& shelf-generation and branch-and-bound until all items are scheduled (see Figure 1). Note that this is a finite process, because in every iteration, the required quantity of at least one item will decrease by at least 1 . 


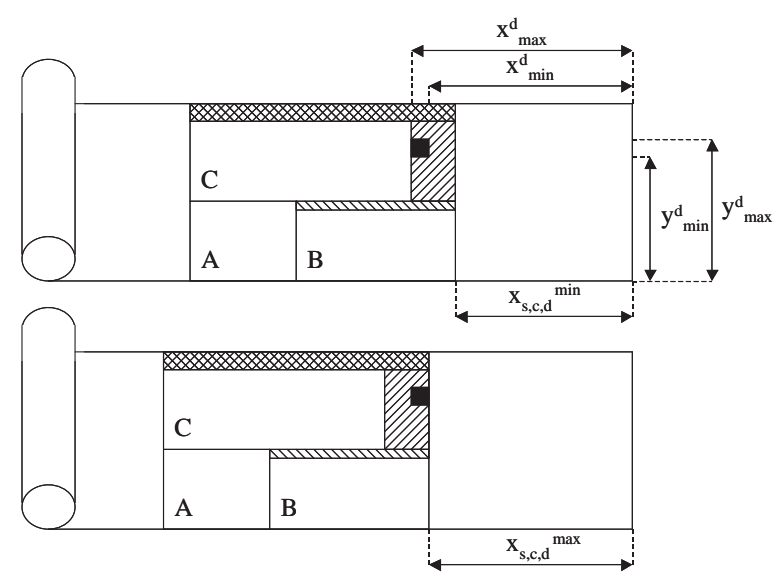

Figure 3: Example shelf with three items

\section{Second stage}

After this first stage, we have determined a set of shelves that meets the quantity requirements. However, the sequence in which these shelves will be cut is still unknown, which means that we are left with a pattern sequencing problem (PSP). In the second stage of the TSH, we will solve this PSP in two phases: first we apply the defects avoiding sequence (DAS) heuristic and afterwards a greedy heuristic. The DAS heuristic determines which shelves will be put in front of the first defect, on top of the first defect, between the first and second defect, and so on. This means that the resulting sequence is not completely fixed (for example, it could be that there are five shelves placed between the first and second defect but their mutual sequence will still be undetermined). This last degree of freedom will be used to minimize the orders-in-progress by using Faggioli and Bentivoglio's greedy heuristic [7] (see also Figure 1). After these two stages, we end up with a solution to the 2D-CSP as encountered at $\mathrm{X}$.

Let us start with the example shelf of Fig. 2, which has been shown twice in Fig. 3. The shelf consists of two bases. In turn, the first base consists of items $A$ and $B$ and the second base is simply equal to item $C$. The hatched areas denote the waste of the shelf. Now let us define three sorts of waste (see also Fig. 2): 


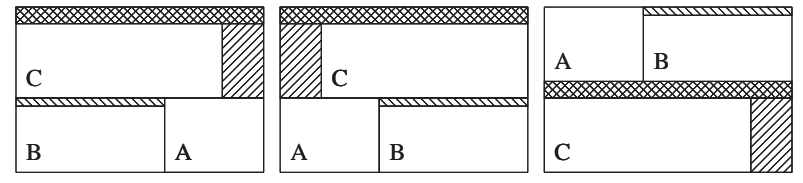

Figure 4: Some configurations of the example shelf

- First order waste - Waste due to the combination of items with different widths within one base (note that the width of an item lies parallel to the $y$-axis, i.e., parallel to the width of fabrics roll).

- Second order waste - Waste due to the combination of bases with different heights within one shelf (note that the height of a base lies parallel to the $x$-axis, i.e., the parallel to the height of the fabrics roll).

- Third order waste - Waste due to the difference between the total width of the bases and the width of the fabrics roll.

The essential assumption for the DAS heuristic to be applicable, is the a priori knowledge of the exact locations of the defects. The main idea of the DAS heuristic is to find a sequence of the shelves selected in stage one, such that we "cut around" the defects, i.e., the defects will be located within the waste pieces. Note that the probability of the existence of a feasible solution depends on the size and number of defects. The DAS heuristic has been created for problems with small size defects (in comparison to the items to be cut) which occur infrequently. Recall from the previous section that we have only created promising bases and shelves. This means that the probability that a defect fits in a piece of first order waste is small in comparison to a piece of second or third order waste. Therefore, the DAS heuristic assumes that this probability is zero, which means that it will not try to fit a defect in a piece of first order waste.

The configuration of a shelf is irrelevent for the waste minimization of the first stage. This means that we can exploit this freedom to find several possibilities of cutting around a defect. Due to the orthogonal guillotine cutting constraint and the assumption that first order waste cannot be used, we only allow the interchanging of items and second order waste within a base, and the interchanging of bases and third order waste within a shelf. Using these interchanging rules, we can obtain 24 different configurations of the example shelf of Figure 3 for which some examples are shown in Figure 4. 
Now let us define $w_{s, c}^{3, \min }$ and $w_{s, c}^{3, \max }$ as the distance from the bottom of the shelf to the bottom and top of the third order waste of configuration $c$ of shelf $s$, respectively. Analogously, let $h_{s, c, b}^{2, \min }$ and $h_{s, c, b}^{2, \max }$ be equal to the distance from the right of the shelf to the right and left of the second order waste of base $b$ within configuration $c$ of shelf $s$, respectively. We have assumed that the exact locations of all defect are known in advance. Furthermore, let the coordinates $\left(x_{\text {min }}^{d}, y_{\text {min }}^{d}\right),\left(x_{\text {max }}^{d}, y_{\text {min }}^{d}\right),\left(x_{\text {max }}^{d}, y_{\text {max }}^{d}\right),\left(x_{\text {min }}^{d}, y_{\text {max }}^{d}\right)$ be the smallest envelope of defect $d$ as shown in Figure 3 (in which the envelope is represented by the black rectangle). Then we can check for each configuration $c$ of shelf $s$ whether it can be used to cut around defect $d$ using the following rules:

1. If $w_{s, c}^{3, \min } \leq y_{\min }^{d}, w_{s, c}^{3, \max } \geq y_{\max }^{d}$ and $h_{s} \geq x_{\max }^{d}-x_{\min }^{d}$ where $h_{s}$ equals to the height of shelf $s$, then it is possible to locate defect $d$ within the piece of third order waste of configuration $c$ of shelf $s$. The minimum and maximum $x$-coordinate of configuration $c$ of shelf $s$ that can be used to cover defect $d$ are then given by: $x_{s, c, d}^{\min }=x_{\text {max }}^{d}-h_{s}$ and $x_{s, c, d}^{\max }=x_{\text {min }}^{d}$.

2. For each base $b$ within shelf $s$, check if $h_{s, c, b}^{2, \max }-h_{s, c, b}^{2, \min } \leq x_{\max }^{d}-x_{\min }^{d}$ and $w_{b} \geq y_{\max }^{d}-y_{\min }^{d}$ with $w_{b}$ the width of base $b$, then it is possible to locate defect $d$ within the piece of waste of the second order of base $b$ of configuration $c$ of shelf $s$. The minimum and maximum $x$-coordinate of configuration $c$ of shelf $s$ that can be used to cover defect $d$ are then given by: $x_{s, c, d}^{\min }=x_{\max }^{d}-h_{s, c, b}^{2, \max }$ and $x_{s, c, d}^{\max }=x_{\min }^{d}-h_{s, c, b}^{2, \min }$.

Figure 3 shows the minimum and maximum possibility of locating the defect within the second order waste of base $C$. Note that there are some other configurations of the example shelf which result in exactly the same minimum and maximum $x$-coordinates. If we have checked all configurations of a shelf, then we will set the minimum and maximum $x$-coordinate of shelf $s$ to cover defect $d$ equal to one with the largest interval. Let $c_{m i n}$ be the configuration with the largest interval, then: $\left(x_{s, d}^{\min }, x_{s, d}^{\max }\right)=\left(x_{s, c_{\min }, d}^{\min }, x_{s, c_{\min }, d}^{\max }\right)$. By doing this we decrease the number of variables of the optimization problem which we will discuss later on in this section. We are aware of the fact that we might also eliminate some high-quality solutions, however by keeping the most promising possibility we hope to limit this effect.

Besides the three sorts of waste we have introduced, we also consider fourth order waste, i.e., waste between two subsequent shelves. If we would place all 
shelves directly next to each other then the fourth order waste would be zero. However, due to the defects this might not always be possible. Let us denote the width of the fourth order waste just before the shelf that covers up defect $d$ as $u^{d}$. Furthermore, let us assume, without loss of generality, that all defect are sorted such that $x_{\text {min }}^{1} \leq x_{\min }^{2} \leq \ldots \leq x_{\min }^{D}$ (with $D$ the number of defects) then a mathematical programming formulation for minimizing fourth order waste can be given as follows:

$$
(P 2): \quad \min \sum_{d=1}^{D} u^{d}+y_{0}^{d} h^{d}
$$

subject to

$$
\begin{gathered}
\sum_{d=1}^{D+1} y_{s}^{d-1, d}+\sum_{d=1}^{D} y_{s}^{d}=1, \quad \forall s \in \mathcal{S} \\
\sum_{s \in \mathcal{S}_{d}} y_{s}^{d}+y_{0}^{d}=1, \quad \forall d \in \mathcal{D} \\
\sum_{i=1}^{d}\left(\sum_{s \in \mathcal{S}} y_{s}^{i-1, i} h_{s}+u_{i}\right)+\sum_{i=1}^{d-1}\left(\sum_{s \in \mathcal{S}_{i}} y_{s}^{i} h_{s}+y_{0}^{i} h^{i}\right) \leq \sum_{s \in \mathcal{S}_{d}} y_{s}^{d} x_{s, d}^{\max }+y_{0}^{d} x_{\text {max }}^{d}, \quad \forall d \in \mathcal{D}(10) \\
\sum_{i=1}^{d}\left(\sum_{s \in \mathcal{S}} y_{s}^{i-1, i} h_{s}+u_{i}\right)+\sum_{i=1}^{d-1}\left(\sum_{s \in \mathcal{S}_{i}} y_{s}^{i} h_{s}+y_{0}^{i} h^{i}\right) \geq \sum_{s \in \mathcal{S}_{d}} y_{s}^{d} x_{s, d}^{\min }+y_{0}^{d} x_{\min }^{d}, \quad \forall d \in \mathcal{D}(11) \\
u_{d} \geq 0, y_{s}^{d-1, d}, y_{0}^{d} \in\{0,1\}, \quad \forall d \in \mathcal{D}, s \in \mathcal{S} \\
y_{s}^{d} \in\{0,1\}, \quad \forall d \in \mathcal{D}, s \in \mathcal{S}_{d}
\end{gathered}
$$

in which $y_{0}^{d}$ equals 1 if and only if none of the shelves is used to cover up defect $d$, i.e., defect $d$ will be "covered" by an empty shelf with height equal to the height of the defect $\left(h^{d}=x_{\max }^{d}-x_{\min }^{d}\right)$. The variable $y_{s}^{d-1, d}$ equals 1 if and only if shelf $s$ will be used in the space between the $(d-1)^{t h}$ and $d^{\text {th }}$ defect and $y_{s}^{d}$ equals 1 if and only if shelf $s$ will be used to cover defect $d$. Furthermore, $\mathcal{D}=\{1,2, \ldots, D\}$ represents the set of defects and $\mathcal{S}_{d} \subseteq \mathcal{S}$ represents the set of shelves that can be used to cover defect $d$.

The objective function (7) minimizes the total fourth order waste under the constraints that all shelves are used exactly once (8) and each defect $d$ is covered 


\begin{tabular}{c|c|c|c|c}
\hline Order & Sub & Width & Height & Quantity \\
\hline \hline 1 & 1 & 1,053 & 2,133 & 1 \\
& 2 & 1,231 & 2,133 & 2 \\
\hline 2 & 1 & 801 & 1,436 & 1 \\
& 2 & 1,456 & 1,732 & 3 \\
& 3 & 911 & 1,241 & 1 \\
\hline 3 & 1 & 1,112 & 2,240 & 2 \\
& 2 & 1,617 & 1,751 & 1 \\
\hline \multirow{2}{*}{4} & 1 & 1,040 & 1,754 & 2 \\
& 2 & 803 & 910 & 1 \\
\hline 5 & 1 & 1,104 & 1,686 & 2 \\
& 2 & 1,557 & 1,918 & 1 \\
\hline
\end{tabular}

Table 1: Order data of the example problem

by a shelf from the corresponding set of shelves $\mathcal{S}_{d}$ or remains uncovered (9). Note that in the latter case the corresponding empty shelf is actually fourth order waste and contributes to the objective function. Constraints (10) state that the sum of the heights of all used shelves plus the total fourth order waste up till the shelf that covers defect $d$ should be less than or equal to the maximum $x$-coordinate of the shelf that covers defect $d$. On the other hand, constraints (11) state that the sum of the heights of all used shelves plus the total fourth order waste up till the shelf that covers defect $d$ should be larger than or equal to the minimum $x$-coordinate of the shelf that covers defect $d$.

We have implemented a standard branch-and-bound scheme to solve the Mixed Integer Programming (MIP) formulation of (7)-(12).

\section{Example}

In this section we will demonstrate the TSH as introduced in the previous sections. Analogous to the theory, we will focus on the first phase of the second stage: the DAS heuristic. Consider the order data as given in Table 1. We have five different customers (orders), each requiring multiple sub orders, which in turn consist of identical items with a certain quantity. Let us assume that these 17 items have to be cut from a roll of fabrics with width 2,500, height 


\begin{tabular}{c|c|c|c|c}
\hline$d$ & $x_{\min }^{d}$ & $x_{\max }^{d}$ & $y_{\min }^{d}$ & $y_{\max }^{d}$ \\
\hline \hline 1 & 10,675 & 10,699 & 1,241 & 1,257 \\
\hline 2 & 12,911 & 12,943 & 2,001 & 2,027 \\
\hline
\end{tabular}

Table 2: Defect data of the example problem

\begin{tabular}{c|c|c|c|c}
\hline$s$ & $h_{s}$ & $W_{s}$ & $b$ & Items \\
\hline \hline 1 & 1,754 & 39,048 & 1 & 2.2 \\
& & & 2 & 4.1 \\
\hline 2 & 1,754 & 39,048 & 1 & 2.2 \\
& & & 2 & 4.1 \\
\hline 3 & 2,133 & 262,393 & 1 & 1.2 \\
& & & 2 & 1.2 \\
\hline 4 & 2,044 & 578,240 & 1 & 5.2 \\
& & & 2 & $2.34 .2 R$ \\
\hline 5 & 1,751 & $1,504,659$ & 1 & 3.2 \\
& & & 2 & 2.1 \\
\hline 6 & 2,224 & 81,054 & 1 & $3.1 R 3.1 R$ \\
\hline 7 & 1,686 & 395,870 & 1 & 5.1 \\
& & & 2 & 5.1 \\
\hline 8 & 2,509 & 492,312 & 1 & $2.2 R 1.1 R$ \\
\hline
\end{tabular}

Table 3: Solution after stage one

25,000 and two defects, from which the exact locations are known in advance and given in Table 2. Furthermore, the fabric allows free rotation of all items, which means that we can omit the rotation constraints (4) and (5) from (P1). Finally, assume that the cutting table restricts the shelves to a maximum height of 3,000 .

Table 3 shows the solution after stage one, in which the addition $R$ means that an item is rotated. Without defects, this solution would result in a trim-loss percentage of $8.58 \%$, however the fabrics roll used contains some. The first step of the DAS heuristic is to determine which shelves can be used to cover up the defects, i.e., determining for each shelf and for each defect the largest interval $\left(x_{s, d}^{\min }, x_{s, d}^{\max }\right)$. Table 4 shows the non-zero intervals. Note that each possibility requires a particular configuration as shown in Figure 5. The small black dots 


\begin{tabular}{c|c|c|c|c}
\hline$d$ & $s$ & $x_{s, d}^{\min }$ & $x_{s, d}^{\max }$ & Waste type \\
\hline \hline 1 & 3 & 8,566 & 10,675 & $3^{\text {th }}$ order \\
& 4 & 10,573 & 10,675 & $2^{\text {nd }}$ order \\
& 7 & 9,013 & 10,675 & $3^{\text {th }}$ order \\
\hline 2 & 4 & 12,817 & 12,911 & $2^{\text {nd }}$ order \\
& 5 & 12,628 & 12,911 & $2^{\text {nd }}$ order \\
\hline
\end{tabular}

Table 4: Possibilities to cover up the defects

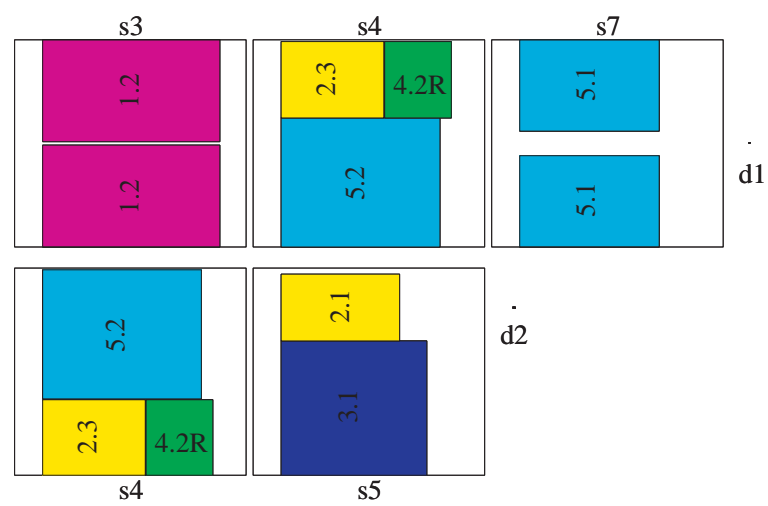

Figure 5: Configurations of the possibilities

$d 1$ and $d 2$ represent the vertical location of the defects. We can see that we can use the third order waste within shelf $s 3$ and $s 7$ to cover up defect $d 1$, i.e., we can horizontally shift both shelves over the full height on top of $d 1$. Shelf 4 can also be used to cover up defect $d 1$, however, we have to use its second order waste to do so.

Now that we have created all possibilities, we will solve the MIP problem $(P 2)$ by branch-and-bound. The results of this step are summarized in Table 5 . Recall that location 0-1 denotes the space between the start of the roll (defect "zero") and the first defect and location 1 denotes the space on top of defect $d 1$. Note that the mutual sequence of the shelves in $0-1$ is still undetermined. This freedom will be used in an attempt to minimize the maximum number of orders in progress by the greedy heuristic in the second phase of the second stage as discussed in Section 2. The final solution is graphically shown in Figure 6 . We can clearly see that the resulting cutting plan "cuts around" the defects. However, the price to pay is the additional fourth order waste (denoted by the 


\begin{tabular}{c|c|c}
\hline Location & $s$ & $u_{d}$ \\
\hline \hline $0-1$ & $1,4,6,8$ & - \\
1 & 3 & 143 \\
$1-2$ & 2 & - \\
2 & 5 & 67 \\
$2-3$ & 7 & - \\
\hline
\end{tabular}

Table 5: Results of the DAS heuristic

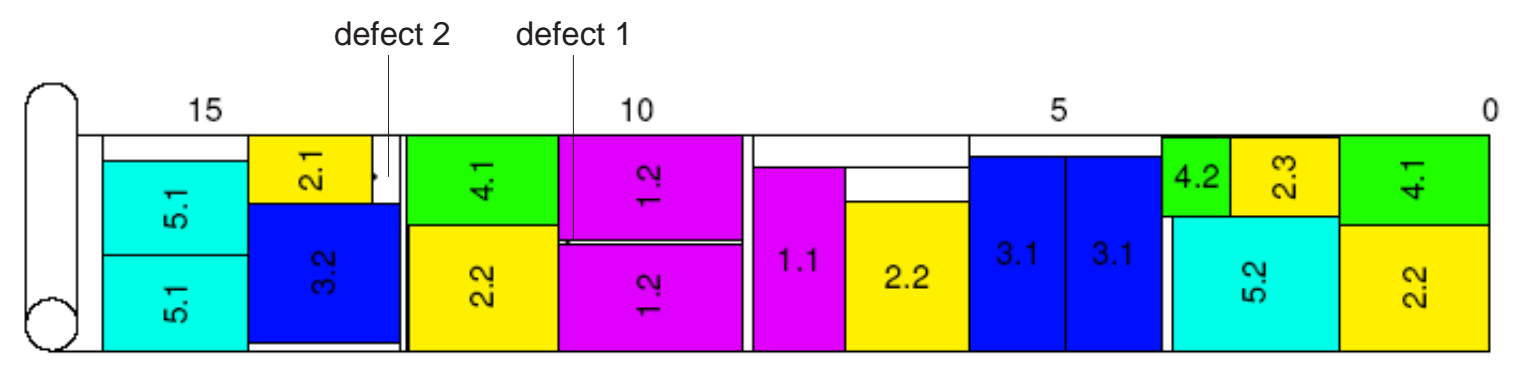

Figure 6: Solution to the example problem

vertical white stripes). The trim-loss percentage of cutting plan is equal to 9.77\%. Recall that the trim-loss percentage after the first stage equals $8.58 \%$, which means that an increase of $1.19 \%$ due to the defects.

\section{$5 \quad$ Practical Results}

We have applied the TSH to the 2D-CSP of the roller blinds production process, i.e., we have computed cutting plans for 101 real-life orders of which we assume that they all allow free rotation of the items. Analogous to the example problem, the fabrics rolls used had a width of 2,000 and a height of 25,000. The cutting table sets a maximum on the height of the shelves of 3,000. Each roll contains zero to five defects, with an average of two. Together, the orders require 210 items which we have split up in batches according to their fabrics color. This split resulted in 16 batches, from which some contain over 20 items and others contain only a few items. The average width and height of the items equals 938 and 1,228 , respectively. In Section 2 we implicitly assumed that the roll 


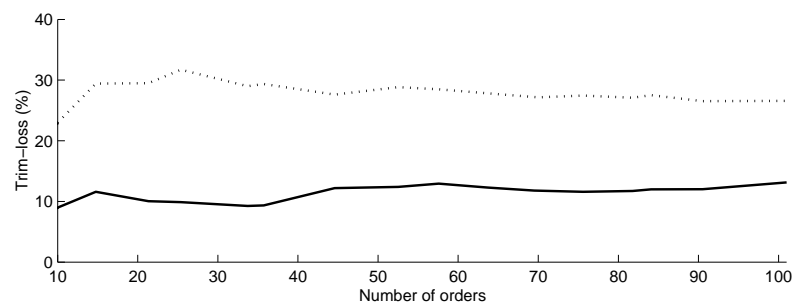

Figure 7: Trim-loss using TSH (solid) and CH (dotted) as function of the number of orders

is always "high enough", i.e., we never reach the end of a roll. In practice, this is certainly not the case. Therefore, we will combine all rolls after each other and "glue" them together with a "height-less" defect over the full width of the roll (thus, $x_{\text {min }}^{d}=25,000, x_{\max }^{d}=25,000, y_{\min }^{d}=0$, and $y_{\text {max }}^{d}=2,000$ ). By doing this, we ensure that none of the shelves will cross two different rolls. Furthermore, we start a batch with the residual roll of the previous batch in order to simulate the fact that, in general, we do not start with a full roll.

The solid line in Figure 7 shows the resulting trim-loss percentage as function of the number of orders after applying the TSH. Initially, the trim-loss percentage of the TSH is relatively low. This suggests that the first batches could relatively easy be combined to a low-waste cutting plan. However, as the number of orders increases, the trim-loss percentage seem to level out at a higher level. After 101 orders, the trim-loss percentage equals $13.2 \%$.

This percentage does not have any meaning unless we compare it with the trimloss percentage that was achieved at $\mathrm{X}$ without our method. One could decide to compare the results of the TSH to the overall trim-loss percentage which can be obtained by dividing the total area of all produced orders by the total area of all used rolls of fabric. However, this would be unfair. In practice, the trim-loss percentage suffers from waste due to all kinds of production failures. Therefore, we have to conclude that we cannot use the general trim-loss percentage for comparison. To make a more fair comparison, we simulate practice with the same orders, the same fabrics and no production failures. Let us call the heuristic that tries to mimic the behavior of the operator at the cutting machine in practice, the current heuristic $(\mathrm{CH})$. This heuristic can be described in five steps: 
1. Pick the first $Y$ items to be cut (assuming that there are at least $Y$ items to be cut, otherwise pick all items).

Although the batch might consist of more than $Y$ items, this step tries to simulate the fact that an operator will only leaf through the first couple of order forms. Any order form contains only one suborder with its corresponding quantity. This means that if, for example, an order consists of two subs then there will be two different forms.

2. Within those first $Y$ items, look for two items which can be cut from a single sheet on top of each other. If such a height combination exists then roll out a piece of fabric with its height to the maximum width of the selected items.

3. If a combination, as described in the previous step, does not exist, then try to combine two items which can be cut from a single sheet rotated on top of each other (if the fabrics allows rotation). If such a width combination exists, then roll out the piece of fabrics with its height equal to the maximum height of the selected items.

4. If a combination, as described in the previous steps, does not exist, then simply the first item and roll out the smallest piece of fabrics from which the item can be cut (rotated or non-rotated).

5. If the unrolled piece of fabrics does not contain any defects, or they can be cut around with the selected item(s), then cut the items. If the unrolled piece of fabrics does contain some defects, which cannot be cut around by the previously selected item(s) but can be cut around by another item from the full batch, then cut that item. Otherwise, cut just after the defect and throw away the resulting piece of fabrics.

In practice, it is certainly not clear how many order forms the operator leafs through to search for combinations of items. In fact, none of the operators will always pick the same number of order forms to examine. It depends on the time-pressure on production and above all on the "day" of the operator. As a result, finding an appropriate value for the fixed input parameter $Y$ is a difficult task. On average, the operators will pick five order forms to examine. As a result, we have set $Y$ equal to five and we have analyzed the sensitivity of the heuristic to this parameter. It turns out that this sensitivity is small ( $\pm 2 \%$ trim-loss variation if we vary $Y$ between 3 and 10) which justifies our assumption. 
The $\mathrm{CH}$ will only search for width and height combinations. The possibility of a cross combination, i.e., a combination of a rotated and non-rotated item will not be considered. Although such solutions could improve the trim-loss percentage, they are ignored in practice since it simply takes too much time to consider all those possibilities.

The dotted line in Fig. 7 represents the resulting trim-loss percentage as function of the number of orders. After 101 orders the average trim-loss percentage is equal to $26.6 \%$. It should be noted that this percentage matches our initial estimate based on the overall trim-loss percentage and a estimation of the trimloss due to production failures. It is clear that our TSH outperforms the current heuristic. In fact, the resulting waste has been reduced by more than $50 \%$ for this example.

These experimental results have been the motivation for the management of $\mathrm{X}$ to go ahead with the implementation of our method in the roller blind production process. Due to the fact that we have to preserve the manufacturer's interests we cannot present the exact numbers after one year of production. However, we are allowed to tell that the implementation has been successful and that the waste (excluding an estimation of the waste due to production failures) has been reduced by $\pm 40-50 \%$.

\section{Concluding remarks}

In this paper we considered a two-dimensional cutting stock problem encountered in the rolling blind production process of a large manufacturer of window covering products. Because of the specific problem characteristics we developed a method, that consists of a combination of adjusted existing and new methods. In particular, we developed the DAS heuristic, to deal with defects in the fabrics. This heuristic tries to sequence the shelves in such a way that the defects end up in waste as much as possible. We feel that this systematic approach of handling defects is the main contribution of our work.

After a comparison of our method with a simulation of "current practice", which indicated that substantial waste reductions could be achieved, management of the manufacturer has approved implementation of our method in one of their factories in the Netherlands, where it has been operational since 2005. The 
actual waste reductions have indeed been substantial and significant cost reductions have been achieved. Implementation of our method in other factories is considered.

\section{References}

[1] J.E. Beasly. Algorithms for unconstrained two-dimensional guillotine cutting. Journal of Operaional Research Society, 36(4):297-306, 1985.

[2] G. Belov and G. Scheithauer. A branch-and-cut-and-price algortihm for one-dimensional stock cutting and two-dimensional two-stage cutting. European Journal of Operational Research, Article in press, 2005.

[3] E.E. Bischoff. Cutting and packing. European Journal of Operations Research, 84(3):503-505, 1995.

[4] P. De Cani. A note on the two-dimensional rectangular cutting-stock problem. Journal of Operational Research Society, 29(7):703-706, 1978.

[5] J.M. Valério de Carvalho. Lp models for bin packing and cutting stock problem. European Journal of Operational Research, 141(2):253-273, 2002.

[6] H. Dyckhoff. A typology of cutting and packing problems. European Journal of Operations Research, 44(2):145-149, 1990.

[7] E. Faggioli and C.A. Bentivoglio. Heuristics and exact methods for the cutting sequencing problem. European Journal of Operational Research, 110(3):564-575, 1998.

[8] P.C. Gilmore and R.E. Gomory. A linear programming approach to the cutting stock problem. Operations Research, 9(6):849-859, 1961.

[9] P.C. Gilmore and R.E. Gomory. A linear programming approach to the cutting stock problem - part ii. Operations Research, 11(6):863-888, 1963.

[10] P.C. Gilmore and R.E. Gomory. Multistage cutting stock problems of two and more dimensions. Operations Research, 13(1):94-120, 1965.

[11] C. Goulimis. Optimal solutions for the cutting stock problem. European Journal of Operational Research, 16(6):1100-1114, 1990. 
[12] S.G. Hahn. On the optimal cutting of defective sheets. Operations Research, 16(6):1100-1114, 1968.

[13] M. Hifi and C. Roucairol. Approximate and exact algorithms for constrained (un) weighted two-dimensional two-staged cutting stock problems. Journal of Combinatorial Optimization, 5(4):465-494, 2001.

[14] K.K. Lai. Developing a simulated annealing algorithm for the cutting stock problem. Computers \&3 Industrial Engineering, 32(1):115-127, 1997.

[15] C.H. Yung T.W. Lueng and M.D. Troutt. Applications of the genetic search and simulated annealing to the two-dimensional non-guillotine cutting stock problem. Computers $\& 3$ Industrial Engineering, 40(3):201-214, 2001.

[16] F. Vanderbeck. A nested decomposition approach to a three-stage, twodimensional cutting-stock problem. Management Science, 47(6):864-879, 2001. 